\title{
An efficient hybrid numerical scheme for solving general second order initial value problems (IVPs)
}

\author{
A. O. Adeniran, B. S. Ogundare * \\ Department of Mathematics, Obafemi Awolowo University, Ile-Ife, 220005, Nigeria \\ *Corresponding author E-mail: ogundareb@yahoo.com, bogunda@oauife.edu.ng
}

Copyright (C)2015 Adeniran and Ogundare. This is an open access article distributed under the Creative Commons Attribution License, which permits unrestricted use, distribution, and reproduction in any medium, provided the original work is properly cited.

\begin{abstract}
The paper presents a one step hybrid numerical scheme with two off grid points for solving directly the general second order initial value problems of ordinary differential equations. The scheme is developed using collocation and interpolation technique. The proposed scheme is consistent, zero stable and of order four. This scheme can estimate the approximate solution at both step and off step points simultaneously by using variable step size. Numerical results are given to show the efficiency of the proposed scheme over the existing schemes.
\end{abstract}

Keywords: Collocation; Consistency; Initial value problems; Interpolation, Region of Absolute stability; Stiff ordinary differential equation; Zero Stability.

\section{Introduction}

The paper considered the approximate solution of general second order initial value problem of the form:

$y^{\prime \prime}=f\left(x, y(x), y^{\prime}(x)\right), \quad y\left(x_{0}\right)=y_{0}, \quad y^{\prime}\left(x_{0}\right)=y_{0}^{\prime}$

where $a \leq x \leq b ; a=x_{0}<x_{1}<x_{2}<\cdots<x_{N-1}=b, N=\frac{b-a}{h}$. $N=0,1 \cdots N-1$ and $h=x_{n+1}-x_{n}$ is called the step length. The condition on the function $f(x, y(x))$ are such that existence and uniqueness of solution is guaranteed(see Wend[17]).

The numerical solution of equation (1) had received lots of attention and is still receiving such an attention due to the fact that many physical sciences and engineering problems formulated into mathematical equation result in the above type.

In most application, equation (1) is solved by reducing it to system of first order ordinary differential equations and appropriate numerical method could be employed to solve the resultant system. The setbacks of this approach had been reported by scholars, among them are Bun and Vasil'yer [5] and Awoyemi et al [4].

The method of collocation and interpolation of the power series approximation to generate continuous linear multistep method has been adopted by many scholars, among them are Fatunla[8], Awoyemi et al.[4], Olabode [13], Vigo-Aguiar and Ramos [16], and Twizel and Khaliq [15], to mention a few. They develop an implicit linear multistep method which are implemented in predictor corrector mode and adopted Taylor's series expansion to provide starting values. In spite of the advantages of linear multistep method, they are usually applied to initial value as a single formula and this has some inherent disadvantages, for instance the implementation of the method in 
predictor-corrector mode are very costly and subroutine are very complicated to write because they require special technique to supply the starting values.

In order to cater for the above mentioned setbacks, scholars such as Jator[10], and Jator and Li[11] come up with the ideal of block method which simultaneous generate approximation at different grid points within the interval of integration. Block method are less expensive in terms of the number evaluations compared to the linear multistep method or Runge-Kutta method, block method does not require predictor or starting values.

In this paper, we proposed a hybrid one step with two off step points method for the solution of general second order initial value problem, we also modified the existing block method to accommodate general second order ordinary differential equation and give conditions for the zero stability of the modified method.

\section{Derivation of the Scheme}

We consider a power series approximate solution in the form:

$y(x)=\sum_{j=0}^{c+i-1} a_{j} x^{j}$

where $\mathrm{c}$ and $\mathrm{i}$ are number of distinct collocation and interpolation points respectively. Substituting the second derivative of equation (2) into equation (1) gives:

$f\left(x, y(x), y^{\prime}(x)\right)=\sum_{j=0}^{c+i-1} j(j-1) a_{j} x^{j-2}$,

we consider a grid point of step length one and off step points at $x=x_{n+\frac{1}{3}}$ and $x=x_{n+\frac{2}{3}}$.

Collocating equation (3) at points $x=x_{n}, x_{n+\frac{1}{3}}, x_{n+\frac{2}{3}}$ and $x_{n+1}$ and interpolating (2) at $x=x_{n}$ and $x_{n+\frac{1}{3}}$ leads to a system of non linear equation of the form:

$$
\left(\begin{array}{cccccc}
1 & x_{n} & x_{n}^{2} & x_{n}^{3} & x_{n}^{4} & x^{5} \\
1 & x_{n+\frac{2}{3}} & x_{n+\frac{2}{3}}^{2} & x_{n+\frac{2}{3}}^{3} & x_{n+\frac{2}{3}}^{4} & x_{n+\frac{2}{3}}^{5} \\
0 & 0 & 2 & 6 x_{n} & 12 x_{n}^{2} & 20 x_{n}^{3} \\
0 & 0 & 2 & 6 x_{n+\frac{1}{3}} & 12 x_{n+\frac{1}{3}}^{2} & 20 x_{n+\frac{1}{3}}^{3} \\
0 & 0 & 2 & 6 x_{n+\frac{2}{3}} & 12 x_{n+\frac{2}{3}}^{2} & 20 x_{n+\frac{2}{3}}^{3} \\
0 & 0 & 2 & 6 x_{n+1} & 12 x_{n+1}^{2} & 20 x_{n+1}^{3}
\end{array}\right)\left(\begin{array}{c}
a_{0} \\
a_{1} \\
a_{2} \\
a_{3} \\
a_{4} \\
a_{5}
\end{array}\right)=\left(\begin{array}{c}
y_{n} \\
y_{n+\frac{2}{3}} \\
f_{n} \\
f_{n+\frac{1}{3}} \\
f_{n+\frac{2}{3}} \\
f_{n+1}
\end{array}\right)
$$

Solving equation (4) for $a_{j}^{\prime}$ s, which are constants to be determined and substituting into equation (2) gives a continuous hybrid multistep method of the form:

$y(x)=\sum_{j=0}^{k} \alpha_{j}(x) y_{n+j}+\sum_{v i} \alpha_{v i} y_{n+v i}+h^{2}\left[\sum_{j=0}^{k} \beta_{j}(x) f_{n+j}+\sum_{v i} \beta_{v i} f_{n+v i}\right]$,

where $k=1, v i=\frac{1}{3}, \frac{2}{3}$, yield the parameter $\alpha_{j}$ and $\beta_{j}, j=0, v i, 1$ as $\alpha_{0}(t)=1-\frac{3}{2} t$

$\alpha_{\frac{2}{3}}=\frac{3}{2} t$

$\beta_{0}=h^{2}\left[\frac{3}{4} t^{4}-\frac{11}{12} t^{3}+\frac{1}{2} t^{2}-\frac{9}{40} t^{5}-\frac{14}{135} t\right]$

$\beta_{\frac{1}{3}}=h^{2}\left[\frac{27}{40} t^{5}-\frac{15}{8} t^{4}+\frac{18}{12} t^{3}-\frac{11}{45} t\right]$

$\beta_{\frac{2}{3}}=h^{2}\left[\frac{3}{2} t^{4}-\frac{27}{40} t^{5}-\frac{3}{4} t^{3}+\frac{1}{45} t\right]$

$\beta_{1}=h^{2}\left[-\frac{3}{8} t^{4}+\frac{1}{6} t^{3}+\frac{9}{40} t^{5}-\frac{1}{135} t\right]$

and 
$t=\frac{x-x_{n}}{h}$

Evaluating the above at $t=1$ and $t=\frac{1}{3}$ gives:

$y_{n+1}=-\frac{1}{2} y_{n}+\frac{3}{2} y_{n+\frac{2}{3}}+h^{2}\left[\frac{1}{216} f_{n}+\frac{1}{18} f_{n+\frac{1}{3}}+\frac{7}{72} f_{n+\frac{2}{3}}+\frac{1}{108} f_{n+1}\right]$

$y_{n+\frac{1}{3}}=\frac{1}{2} y_{n}+\frac{1}{2} y_{n+\frac{2}{3}}+h^{2}\left[-\frac{1}{216} f n-\frac{5}{108} f_{n+\frac{1}{3}}-\frac{1}{216} f_{n+\frac{2}{3}}\right]$

note also that $\frac{d t}{d x}=\frac{1}{h}$

Differentiating (5) yields:

$y^{\prime}(x)=\frac{1}{h} \sum_{j=0}^{k} \alpha_{j}^{\prime}(x) y_{n+j}+\frac{1}{h} \sum_{v i} \alpha_{v i}^{\prime} y_{n+v i}+h\left[\sum_{j=0}^{k} \beta_{j}^{\prime}(x) f_{n+j}+\sum_{v i} \beta_{v i}^{\prime} f_{n+v i}\right]$,

where $\alpha_{0}^{\prime}(t)=-\frac{3}{2 h}$

$\alpha_{\frac{2}{3}}^{\prime}(t)=\frac{3}{2 h}$

$\beta_{0}^{\prime}(t)=h\left[3 t^{3}-\frac{33}{12} t^{2}+t-\frac{45}{40} t^{4}-\frac{14}{135}\right]$

$\beta_{\frac{1}{3}}^{\prime}(t)=h\left[\frac{27}{8} t^{4}-\frac{15}{2} t^{3}+\frac{18}{4} t^{2}-\frac{11}{45}\right]$

$\beta_{\frac{2}{3}}^{\prime}(t)=h\left[6 t^{3}-\frac{27}{8} t^{4}-\frac{9}{4} t^{2}+\frac{1}{45}\right]$

$\beta_{1}^{\prime}(t)=h\left[-\frac{3}{2} t^{3}+\frac{1}{2} t^{2}+\frac{9}{8} t^{4}-\frac{1}{135}\right]$

On evaluating equation(8) above at $t=0, \frac{1}{3}, \frac{2}{3}$ and 1 , we have

$$
\begin{aligned}
h y_{n}^{\prime}-\frac{3}{2} y_{n+\frac{2}{3}}+\frac{3}{2} y_{n} & =h^{2}\left[-\frac{4}{135} f_{n}-\frac{11}{45} f_{n+\frac{1}{3}}+\frac{1}{45} f_{n+\frac{2}{3}}-\frac{1}{135} f_{n+1}\right] \\
h y_{n+\frac{1}{3}}^{\prime}-\frac{3}{2} y_{n+\frac{2}{3}}+\frac{3}{2} y_{n} & =h^{2}\left[\frac{23}{1080} f_{n}+\frac{7}{360} f_{n+\frac{1}{3}}-\frac{17}{360} f_{n+\frac{2}{3}}+\frac{7}{1080} f_{n+1}\right] \\
h y_{n+\frac{2}{3}}^{\prime}-\frac{3}{2} y_{n+\frac{2}{3}}+\frac{3}{2} y_{n} & =h^{2}\left[\frac{1}{135} f_{n}+\frac{1}{5} f_{n+\frac{1}{3}}+\frac{2}{15} f_{n+\frac{2}{3}}-\frac{1}{135} f_{n+1}\right] \\
h y_{n+1}^{\prime}-\frac{3}{2} y_{n+\frac{2}{3}}+\frac{3}{2} y_{n} & =h^{2}\left[\frac{23}{1080} f_{n}+\frac{47}{360} f_{n+\frac{1}{3}}+\frac{143}{360} f_{n+\frac{2}{3}}+\frac{127}{1080} f_{n+1}\right]
\end{aligned}
$$

\section{Modified block method}

According to Chu and Hamilton [6], a general k block, r-point block method is a matrix of finite difference equation of the form

$Y_{m}=\sum_{j=1}^{k} A_{i} Y_{m-i}+h \sum_{i=0}^{k} B_{i} F_{m-i}$,

where all the $A_{i}^{\prime}$ s and $B_{i}^{\prime}$ s are properly chosen $r \times r$ matrix coefficients and $m=0,1,2, \cdots$ represents the block number, $n=m r$ is the first step number of the mth block and $\mathrm{r}$ is the proposed block size.

In order to evaluate the unknown parameter independently, there is need to modify the existing method proposed by Chu and Hamilton [6]. The modified block method is of the form

$A^{0} h^{\lambda} Y_{m}^{(n)}=h^{\lambda} \sum_{i=0}^{k} A^{(i)} Y_{m-i}^{(n)}+h^{\lambda} \sum_{i=1}^{k} B^{(i)} F_{m-i}$ 
where $\mathrm{n}$ is the power of the derivative, $\mu$ is the order of the differential equation. $A^{0}$ and $A^{(i)}$ are $R \times R$ identity and $\lambda$ is the power of $h$ relative to the derivative of the differential equation. Also

$$
\begin{aligned}
& h^{\lambda} Y_{m}^{(n)}=\left[y_{n+\frac{1}{3}}, y_{n+\frac{2}{3}}, \cdots, y_{n+1}, \cdots, h y_{n+\frac{1}{3}}^{\prime}, \cdots, y_{n+1}^{\prime}, \cdots h^{2} y_{n+y_{n+\frac{1}{3}}}^{\prime \prime}, \cdots, h^{2} y_{n+1}^{\prime \prime}, \cdots, h^{n} y_{n+m}^{n}\right]^{T} \\
& h^{\lambda} Y_{m-i}^{(n)}=\left[y_{n-1}, y_{n-2}, \cdots, y_{n}, h y_{n-1}^{\prime}, \cdots, h y_{n}^{\prime}, h^{2} y_{n-1}^{\prime \prime}, h^{2} y_{n-2}^{\prime \prime}, \cdots, h^{m} y_{n}^{m}\right]^{T} \\
& F_{m-i}=\left[f_{n-\frac{1}{3}}, f_{n-\frac{2}{3}}, f_{n-1}, \cdots, f_{m}, f_{n+\frac{1}{3}}, f_{n+\frac{2}{3}}, f_{n+1}, \cdots f_{m}\right]^{T}
\end{aligned}
$$

Evaluating equations (6), (7) and (9) for $h^{\lambda} y_{n+m}^{\lambda}, m=0, \frac{1}{3}, \frac{2}{3}, 1$, gives

$A^{0}=\left(\begin{array}{cccccc}1 & 0 & 0 & 0 & 0 & 0 \\ 0 & 1 & 0 & 0 & 0 & 0 \\ 0 & 0 & 1 & 0 & 0 & 0 \\ 0 & 0 & 0 & 1 & 0 & 0 \\ 0 & 0 & 0 & 0 & 1 & 0 \\ 0 & 0 & 0 & 0 & 0 & 1\end{array}\right), \quad A^{i}=\left(\begin{array}{cccccc}0 & 0 & 0 & 1 & 0 & \frac{1}{3} \\ 0 & 0 & 0 & 1 & 0 & \frac{2}{3} \\ 0 & 0 & 0 & 0 & 0 & 1 \\ 0 & 0 & 0 & 0 & 0 & 1 \\ 0 & 0 & 0 & 0 & 0 & 1 \\ 0 & 0 & 0 & 0 & 0 & 0\end{array}\right), \quad B^{i}=\left(\begin{array}{ccccc}\frac{97}{3240} & \frac{19}{540} h^{2} & -\frac{13}{1080} h^{2} & \frac{1}{405} h^{2} \\ \frac{28}{405} & \frac{22}{135} h^{2} & -\frac{2}{135} h^{2} & \frac{2}{405} h^{2} \\ \frac{13}{120} & \frac{3}{10} h^{2} & \frac{3}{40} h^{2} & \frac{1}{60} h^{2} \\ \frac{1}{8} & \frac{19}{72} h & -\frac{5}{72} h & \frac{1}{72} h \\ \frac{1}{9} & \frac{4}{9} h & \frac{1}{9} h & 0 \\ \frac{1}{8} & \frac{3}{8} h & \frac{3}{8} h & \frac{1}{8} h\end{array}\right)$

Substituting $A^{0}, A^{i}$ and $B^{i}$ into equation (11) gives

$$
\begin{aligned}
& y_{n+\frac{1}{3}}=y_{n}+\frac{1}{3} h y_{n}^{\prime}+h^{2}\left[\frac{97}{3240} f_{n}+\frac{19}{540} f_{n+\frac{1}{3}}-\frac{13}{2304} f_{n+\frac{2}{3}}+\frac{1}{405} f_{n+1}\right] \\
& y_{n+\frac{2}{3}}=y_{n}+\frac{2}{3} h y_{n}^{\prime}+h^{2}\left[\frac{28}{405} f_{n}+\frac{22}{135} f_{n+\frac{1}{3}}-\frac{2}{135} f_{n+\frac{2}{3}}+\frac{2}{405} f_{n+1}\right] \\
& y_{n+1}=y_{n}+h y_{n}^{\prime}+h^{2}\left[\frac{13}{120} f_{n}+\frac{3}{10} f_{n+\frac{1}{3}}+\frac{3}{40} f_{n+\frac{2}{3}}+\frac{1}{60} f_{n+1}\right] \\
& y_{n+\frac{1}{3}}^{\prime}=y_{n}^{\prime}+h\left[\frac{1}{8} f_{n}+\frac{9}{72} f_{n+\frac{1}{3}}-\frac{5}{72} f_{n+\frac{2}{3}}+\frac{1}{72} f_{n+1}\right] \\
& y_{n+\frac{2}{3}}^{\prime}=y_{n}^{\prime}+h\left[\frac{1}{9} f_{n}+\frac{4}{9} f_{n+\frac{1}{3}}+\frac{1}{9} f_{n+\frac{2}{3}}\right] \\
& y_{n+1}^{\prime}=y_{n}^{\prime}+h\left[\frac{1}{8} f_{n}+\frac{3}{8} f_{n+\frac{1}{3}}+\frac{3}{8} f_{n+\frac{2}{3}}+\frac{1}{8} f_{n+1}\right]
\end{aligned}
$$

\section{Analysis of the Scheme}

In this section, we analyze the derived scheme in (12) which includes the order and error constant, Consistency zero stability, and convergence of the method.

\subsection{Order and error constant}

We adopted the method proposed by Fatunla [8] and Lambert [12] to obtain the order of our scheme as $(4,4,4,4,4)^{T}$ and error constant as $\left(-\frac{281}{524880}, \frac{389}{33592320},-\frac{1}{12960},-\frac{76667}{546750000}, \frac{619}{5598720},-\frac{1}{6480}\right)^{T}$

\subsection{Consistency}

According to Gurjinder et al [7]. A linear multistep method is said to be consistent if it has an order of convergence, say $p \geq 1$. Thus, our derived schemes are consistent, since the order is four.

\subsection{Zero Stability}

To obtain the zero stability of the method we consider the following conditions:

(i) The block (11) is said to be stable if as $h \rightarrow 0$ the roots $r_{j}, \mathrm{j}=1(1) \mathrm{k}$ of the first characteristics polynomial $\rho(R)=0$, that is $\rho(R)=\operatorname{det}\left[\sum A^{(i)} R^{k-1}\right]=0$, satisfy $|R| \leq 1$ and for those roots with $|R| \leq 1$, must have multiplicity equal to unity(See Fatunla [8]for details).

(ii) If block (11) be an $R \times R$ matrix then, it is zero stable if as $h^{\mu} \rightarrow 0,\left|R A^{0}-A^{i}\right|=R^{r-\mu}=0$. For those root with $\left|R_{j}\right| \leq 1$, the multiplicity must not exceed the order of the differential equation. 
For our method

$$
\lambda A^{0}-A^{i}=\left(\begin{array}{cccccc}
\lambda & 0 & 0 & -1 & 0 & -\frac{1}{3} \\
0 & \lambda & 0 & -1 & 0 & -\frac{2}{3} \\
0 & 0 & \lambda & 0 & 0 & -1 \\
0 & 0 & 0 & \lambda & 0 & -1 \\
0 & 0 & 0 & 0 & \lambda & -1 \\
0 & 0 & 0 & 0 & 0 & \lambda-1
\end{array}\right)
$$

As $h \rightarrow 0$ in equation (11), (13) reduces to

$\left(\begin{array}{ccc}\lambda & 0 & -1 \\ 0 & \lambda & -1 \\ 0 & 0 & \lambda-1\end{array}\right)$

the determinant of which yields the values $\lambda=0,0,1$. Taking the determinant of (13) we have $\lambda^{5}(\lambda-1)=0$, which gives $\lambda=0$ or $\lambda=1$.

Since the two conditions are satisfied, we conclude that the block method are zero stable .

\subsection{Convergence}

The convergence of our continuous implicit hybrid one step is considered in the light of the fundamental theorem of Dahlquist(Henrici, [9]. we state Dahlquist's theorem without proof.

Theorem 4.1 The neccessary and sufficient condition for a linear multistep to be convergent is for it to be consistent and zero stable

Since our block method is consistent and zero stable, hence our block method is convergent.

\subsection{Region of Absolute Stability of the main method}

The stability polynomial of the method is given by

$\pi(r, \bar{h})=\rho(r)-\bar{h} \delta(r)=0$

where $\bar{h}=\lambda^{2} h^{2}$ and $\lambda=\frac{d f}{d y}$ are assumed constant.

The boundary locus curve is then obtained by putting

$\bar{h}(r)=\frac{\rho(r)}{\delta(r)}$, where $r=e^{i \theta}=\cos \theta+i \sin \theta, \quad 0 \leq \theta \leq \pi$.

The mirror of the equation (15) is then generated through the real axis to obtain the interval of absolute stability of the method.

Table 1: The boundaries of the region of absolute stability of the method

\begin{tabular}{cccccccc}
\hline$\theta^{0}$ & 0 & 30 & 60 & 90 & 120 & 150 & 180 \\
$\bar{h}(\theta)$ & 0 & 47.76 & 15.94 & -194.41 & 128.14 & -1.00 & 224.25 \\
\hline
\end{tabular}

The region of absolute stability of the method is between $\left(\begin{array}{ll}-194.41 & 224.25\end{array}\right)$

\section{Numerical implementation of the scheme}

In this section, we test the effectiveness and validity of our newly derived scheme in equation (12) by applying it to some second order differential equations. All calculations and programs are carried out with the aid of Maple 13 software.

\section{Example 1}

We consider the initial value problem

$y^{\prime \prime}(x)=\frac{\left(y^{\prime}\right)^{2}}{2 y}-2 y, y\left(\frac{\pi}{6}\right)=\frac{1}{4}$ and $y^{\prime}\left(\frac{\pi}{6}\right)=\frac{\sqrt{3}}{2}$

with exact solution given as $y=\sin ^{2} x$. 
Table 2: Numerical result for problem 1 with $h=0.01$

\begin{tabular}{ccccc}
\hline $\mathrm{x}$ & Exact & Numerical & Error & Error in $[2]$ \\
\hline 0.1 & 0.0099667110793792 & 0.0099667110782712 & $1.114 \times 10^{-12}$ & \\
0.2 & 0.0394695029985574 & 0.0394695029786944 & $1.990 \times 10^{-11}$ & \\
0.3 & 0.0873321925451611 & 0.0873321924526160 & $9.255 \times 10^{-11}$ & \\
0.4 & 0.1516466453264171 & 0.1516466452810910 & $4.533 \times 10^{-11}$ & \\
0.5 & 0.2298488470659300 & 0.2298488470170830 & $4.884 \times 10^{-11}$ & \\
0.6 & 0.3188211227616632 & 0.3188211226734520 & $8.821 \times 10^{-11}$ & $1.013 \times 10^{-08}$ \\
0.7 & 0.4150164285498790 & 0.4150164284855940 & $6.429 \times 10^{-11}$ & $4.782 \times 10^{-08}$ \\
0.8 & 0.5145997611506450 & 0.5145997611346470 & $1.599 \times 10^{-11}$ & $1.109 \times 10^{-07}$ \\
0.9 & 0.6136010473465430 & 0.6136010473305330 & $1.601 \times 10^{-10}$ & $1.892 \times 10^{-07}$ \\
1.0 & 0.7080734182735720 & 0.7080734182628380 & $1.071 \times 10^{-10}$ & $1.196 \times 10^{-07}$ \\
1.1 & 0.7942505586276720 & 0.7942505583334210 & $2.943 \times 10^{-10}$ & $3.019 \times 10^{-07}$ \\
1.2 & 0.8686968577706220 & 0.8686968480836530 & $9.687 \times 10^{-09}$ & $2.561 \times 10^{-07}$ \\
1.3 & 0.9284443766844740 & 0.9284443728400360 & $3.844 \times 10^{-09}$ & $1.435 \times 10^{-07}$ \\
1.4 & 0.9711111703343290 & 0.9711111632232120 & $7.111 \times 10^{-09}$ & $1.019 \times 10^{-07}$ \\
1.5 & 0.9949962483002220 & 0.9949962433039740 & $4.996 \times 10^{-09}$ & $2.319 \times 10^{-07}$ \\
1.6 & 0.9991473878973760 & 0.9991473864234970 & $1.474 \times 10^{-09}$ & $5.892 \times 10^{-07}$ \\
1.7 & 0.9833990962897310 & 0.9833990922987680 & $3.990 \times 10^{-09}$ & $1.013 \times 10^{-06}$ \\
1.8 & 0.9483792081670730 & 0.9483792067878650 & $1.379 \times 10^{-09}$ & $1.211 \times 10^{-06}$ \\
1.9 & 0.8954838559572070 & 0.8954838011186470 & $5.484 \times 10^{-08}$ & $1.287 \times 10^{-06}$ \\
2.0 & 0.8268218104318070 & 0.8268217836099970 & $2.682 \times 10^{-08}$ & $1.435 \times 10^{-06}$ \\
\hline
\end{tabular}

\section{Example 2}

We consider the initial value problem

$y^{\prime \prime}(x)-x\left(y^{\prime}\right)^{2}=0, y(0)=1$ and $y^{\prime}(0)=\frac{1}{2}, 0 \leq x \leq 1$

with exact solution given as $y=1+\frac{1}{2} \ln \left(\frac{2+x}{2-x}\right)$.

Table 3: Numerical result for Problem 2 with $h=0.1$

\begin{tabular}{ccccc}
\multicolumn{6}{c}{ Table 3: Numerical result for Problem 2 with $h=0.1$} \\
\hline 0.1 & 1.05004172927849 & 1.05004172927845 & $4.173 \times 10^{-14}$ & $1.144 \times 10^{-10}$ \\
0.2 & 1.10033534773107 & 1.10033534773097 & $1.003 \times 10^{-13}$ & $5.873 \times 10^{-09}$ \\
0.3 & 1.15114043593647 & 1.15114043593637 & $1.001 \times 10^{-13}$ & $1.328 \times 10^{-08}$ \\
0.4 & 1.20273255405408 & 1.20273255405381 & $2.733 \times 10^{-13}$ & $2.318 \times 10^{-08}$ \\
0.5 & 1.25541281188300 & 1.25541281188246 & $5.413 \times 10^{-13}$ & $3.213 \times 10^{-08}$ \\
0.6 & 1.30951960420311 & 1.30951960420216 & $9.519 \times 10^{-13}$ & $6.871 \times 10^{-08}$ \\
0.7 & 1.36544375427140 & 1.36544375427103 & $3.654 \times 10^{-12}$ & $1.012 \times 10^{-07}$ \\
0.8 & 1.42364893019360 & 1.42364893019124 & $2.365 \times 10^{-12}$ & $1.231 \times 10^{-07}$ \\
0.9 & 1.48470027859405 & 1.48470027858920 & $4.847 \times 10^{-12}$ & $2.019 \times 10^{-07}$ \\
1.0 & 1.54930614433406 & 1.54930614432857 & $5.493 \times 10^{-12}$ & $2.991 \times 10^{-07}$ \\
\hline
\end{tabular}

\section{Example 3}

Consider the Scalar test test equation

$y^{\prime \prime}=-\omega^{2} y, \quad y(0)=1$ and $y^{\prime}(0)=0$,

with the exact solution given as $y=\cos (\omega x)$.

Set $\omega=10$

Table 4: Numerical result for Problem 3 with $h=\frac{\pi}{200}$

\begin{tabular}{ccccc}
\hline $\mathrm{x}$ & Exact & Numerical & Error & Error in [3] \\
\hline $5 \pi$ & 1.00000000000000 & 0.999999999973525 & $2.6475 \times 10^{-11}$ & $2.3659 \times 10^{-04}$ \\
$10 \pi$ & 1.00000000000000 & 0.999999999892784 & $1.0722 \times 10^{-10}$ & $5.1547 \times 10^{-04}$ \\
$15 \pi$ & 1.00000000000000 & 0.999999999757807 & $2.4219 \times 10^{-10}$ & $6.2689 \times 10^{-04}$ \\
$20 \pi$ & 1.00000000000000 & 0.999999999757807 & $2.4219 \times 10^{-10}$ & $8.3654 \times 10^{-04}$ \\
\hline
\end{tabular}




\section{Example 4}

We consider the Stiffel and Bettis Problem given by Psihoyios and Simos [14]

$y^{\prime \prime}+y=0.001 \cos (x), \quad y(0)=1, \quad y^{\prime}(0)=0$

$z^{\prime \prime}+z=0.001 \sin (x), \quad y(0)=0, \quad y^{\prime}(0)=0.9995$

Exact Solution

$y(x)=\cos (x)+0.0005 \sin (x), \quad z(x)=\sin (x)-0.0005 \cos (x)$

Table 5: Numerical result for Problem 4 with $h=\frac{1}{320}$

\begin{tabular}{ccc}
\hline $\mathrm{x}$ & $\mathrm{y}$ computed & $\mathrm{z}$ computed \\
\hline$\frac{1}{320}$ & 0.999995122082805 & 0.0031234324216293 \\
$\frac{3}{320}$ & 0.999956098979608 & 0.0093701753784351 \\
$\frac{6}{320}$ & 0.999824399721860 & 0.0187395280367614 \\
$\frac{9}{320}$ & 0.999604913790212 & 0.0281072353266269 \\
$\frac{12}{320}$ & 0.999297660455965 & 0.0374724747441467 \\
$\frac{15}{320}$ & 0.998902666696572 & 0.0468344240021194 \\
$\frac{18}{320}$ & 0.998419967193269 & 0.0561922611022260 \\
$\frac{21}{320}$ & 0.997849604328040 & 0.0655451644072030 \\
$\frac{24}{320}$ & 0.997191628179883 & 0.0748923127129838 \\
\hline
\end{tabular}

Table 6: Absolute errors with $h=\frac{1}{320}$ for problem 4

\begin{tabular}{ccc}
\hline $\mathrm{x}$ & Error in y computed & Error in z computed \\
\hline$\frac{1}{320}$ & $8.5250 \times 10^{-12}$ & $2.6041 \times 10^{-13}$ \\
$\frac{3}{320}$ & $2.5573 \times 10^{-11}$ & $9.4101 \times 10^{-13}$ \\
$\frac{6}{320}$ & $5.1130 \times 10^{-11}$ & $2.3620 \times 10^{-12}$ \\
$\frac{9}{320}$ & $7.6654 \times 10^{-11}$ & $4.2602 \times 10^{-12}$ \\
$\frac{12}{320}$ & $1.0215 \times 10^{-10}$ & $6.6377 \times 10^{-12}$ \\
$\frac{15}{320}$ & $1.2760 \times 10^{-10}$ & $9.4932 \times 10^{-12}$ \\
$\frac{18}{320}$ & $1.5301 \times 10^{-10}$ & $1.2826 \times 10^{-11}$ \\
$\frac{21}{320}$ & $1.7836 \times 10^{-10}$ & $1.6636 \times 10^{-11}$ \\
$\frac{24}{320}$ & $2.0365 \times 10^{-10}$ & $2.0922 \times 10^{-11}$ \\
\hline
\end{tabular}

\section{Example 5}

We consider a highly stiff problem

$y^{\prime \prime}+1001 y^{\prime}+1000 y, \quad y(0)=1, \quad y^{\prime}(0)=-1$

Exact Solution

$y(x)=\exp (-x)$

Table 7: Numerical result for Problem 5 with $h=0.1$

\begin{tabular}{ccccc}
\hline $\mathrm{x}$ & Exact & Numerical & Error & Error in [1] \\
\hline 0.1 & 0.9048374154731 & 0.9048374180155 & $2.05 \times 10^{-11}$ & \\
0.2 & 0.8187307355623 & 0.8187307530341 & $4.39 \times 10^{-11}$ & $5.90 \times 10^{-10}$ \\
0.3 & 0.7408181238342 & 0.7408182206162 & $6.55 \times 10^{-11}$ & \\
0.4 & 0.6703195339539 & 0.6703200459518 & $8.38 \times 10^{-11}$ & $1.20 \times 10^{-09}$ \\
0.5 & 0.6065279785945 & 0.6065306596140 & $9.86 \times 10^{-11}$ & \\
0.6 & 0.5487976280186 & 0.5488116359840 & $1.10 \times 10^{-10}$ & $1.80 \times 10^{-09}$ \\
0.7 & 0.4965121474968 & 0.4965853036729 & $1.19 \times 10^{-10}$ & \\
0.8 & 0.4489469435566 & 0.4493289639928 & $1.24 \times 10^{-10}$ & $1.80 \times 10^{-09}$ \\
0.9 & 0.4045747914741 & 0.4065696596124 & $1.28 \times 10^{-10}$ & \\
1.0 & 0.3574624994369 & 0.3678794410413 & $1.30 \times 10^{-10}$ & $1.80 \times 10^{-09}$ \\
\hline
\end{tabular}




\section{Discussion of Result}

A new one-step continuous block hybrid method with two non-step points of order 4 is proposed for the direct solution of second order. The main method and the additional methods were obtained from the same continuous method derived via interpolation and collocation procedures. The stability properties and region of the method are also discussed. The methods are then applied in block form as simultaneous numerical integrators over nonoverlapping interval. In Tables 2, 3, 4, and 7, we compared the accuracy of proposed method with those Abhulimen and Okunuga[1], Alabi et. al [2], and Ali Shorki [3], . From the tables, it can be observed that the new scheme displayed better accuracy.

\section{Conclusion}

The one step hybrid numerical scheme generated in this paper is accurate, efficient and can compete favorably with existing schemes.

\section{References}

[1] C. E. Abhulimen and S. A. Okunuga (2008): Exponentially Fitted Second Derivative Multistep Method for Stiff Initial Value Problem for ODEs, journal of Engineering science and Applications, 5, 36-49 .

[2] M. O. Alabi, A. T. Oladipo and A. O. Adesanya (2008): Initial Value solvers for Second Order Ordinary Differential Equations Using Chebyshev Polynomial as Basis Functions, Journal of Modern Mathematics, 2(1), 18-27.

[3] Ali Shorki (2012): The Symmetric P-Stable Hybrid Obrenchkoff Methods for the numerical solution of second Order IVPS. J. Pure.Appl. Math. 5(1), 28-35.

[4] D. O. Awoyemi, A. O. Adesanya and S. N. Ogunyebi(2009): Construction of Self Starting Numerov Method for the Solution of Initial Value Problem of General Second Order Ordinary Differential Equation. Journ. Num. math 4(2), $267-278$.

[5] R. A. Bun and Y.D. Vasil'Yev (1992): A Numerical Method for Solving Differential Equations of any Orders. Comp. Math. Phys., 32(3), 317-330.

[6] M. T. Chu and H. Hamilton (1987): Parallel Solution of Ordinary Differential Equations by Multiblock Methods. SIAM Journal of Scientific and Statistical Computations, 8, 342-553.

[7] Gurjinder Singh, V. Kanwar and Saurabh Bhatia (2013): Exponentially fitted variants of the two-step Adams-Bashforth method for the numerical integration of initial value problem. journal of application and applied mathematics, 8(2), $741-755$

[8] S. O. Fatunla (1991): Block methods for second order IVPs, Int.J. Comput.Maths., 41 55-63.

[9] P. Henrici (1962): Discrete Variable Methods in ODE. New York: John Wiley and Sons.

[10] S. N. Jator (2007): A sixth order linear multistep method for direct solution second order differential equation. International journal of pure and applied Mathematics 40(1), 457-472.

[11] S. N. Jator and Li, J. (2009): a self starting linear multistep method for a direct solution of the general second order initial value problems. Intern J. Comp. Math. 86(5), 827-836.

[12] J.D. Lambert (1973): Computational Methods in ODEs. New York: John Wiley.

[13] B. T. Olabode (2009): An accurate scheme by block method for the third order ordinary differential equation. Pacific journal of science and technology. 10(1) http:/www.okamaiuniversity.us/pjst.htm.

[14] G. Psihoyios and T. E. Simos (2003): Trigonometrically fitted predictor-corrector methods for IVPs with Oscillating solution. Journal of Computational and Applied Mathematics, 158 (2003) 135-144.

[15] E. H. Twizel and A. Q. M. Khaliq (1984): Mutiderivative Methods for Periodic IVPs. SIAM Journal of Numerical Analysis, 21, 111-121.

[16] J. Vigo-Aguilar and H. Ramos (2006): Variable Stepsize Implementation of Multistep Methods for y" = f(x, y, y'). Journal of Computational and Applied Mathematics, 192, 114-131. 
[17] V. V. Wend (1969): Existence and Uniqueness of solution of ordinary differential equation. proceedings of the American Mathematical Society. 23(1), 23-27.

[18] Y.A. Yahaya and A. M. Badmus (2009): A Class of Collocation Methods for General Second Order Ordinary Differential Equations. African Journal of Mathematics and Computer Science Research, 2(04), 069-072 . 